\title{
Calculation of Transmittance of Superconductor-Dielectric Periodic Structure
}

\author{
J P Pandey \\ M L K P G College, Balrampur (Up)-271201, India
}

\begin{abstract}
Transfer matrix method (TMM) is used to study the optical properties of photonic crystals (PCs) with super conducting constituent for a stratified medium. The filtering properties in superconductor-dielectric photonic band gap material can be studied by reflectance and transmittance at different incidence angles and temperatures. Here, I have calculated the dispersion and transmittance spectrum of superconductor-dielectric periodic structure based on the transcendental equations derived from TMM and Bloch theorem.
\end{abstract}

Date of Submission: 20 -07-2017

Date of acceptance: $31-07-2017$

\section{INTRODUCTION}

Much work has been done on the computation of the band structures of electromagnetic wave propagation in one-, two- and three-dimensional dielectric periodic structure [1-4]. It has already been proposed that Photonic Crystals (PCs) constructed with ferroelectric or ferromagnetic materials could be tuned by means of an external electric or magnetic field [5]. Recently, it was proposed that photonic crystal of the inverse opal type doped with a liquid crystal [6] and photonic crystals made of semiconductor constituents are suitable to constitute tunable photonic crystal [7]. The idea of the former is based on liquid crystal dielectric response to the external electric field, while the latter is mainly due to the variations of the concentration of free carriers within the semiconductor by means of change in temperature or in the density of dopants.

To foresee novel applications and interesting possibilities of incorporating the photonic crystals concept into superconducting devices, it is interesting to investigate new possibilities of merging new areas of electronics and photonics. The study of photonic band gap consisting of a superconducting material and a dielectric has been reported [8-12]. The gap size is characterized by polarization, penetration depth, and highly dependent on temperature at the vicinity of superconducting transition temperature. The analysis shows that the property of thin photonic crystals may have application in optical region if extremely low relaxation time superconductor is used. This may be asset for superconducting electronics-photonic integration.

\section{Theoretical formulation}

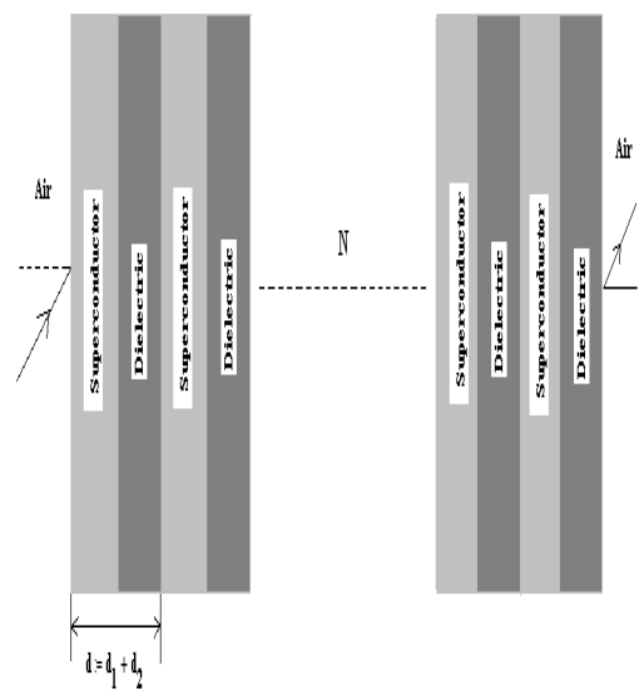

Figure 1: Schematic presentation of superconductor/dielectric periodic structure.

The refractive index profile of the structure is given by

$n(x)= \begin{cases}n_{1}, & 0<x<d_{1} \\ n_{2}, & d_{2}<x<d\end{cases}$

with $\mathrm{n}(\mathrm{x})=\mathrm{n}(\mathrm{x}+\mathrm{d})$ where $\mathrm{n}_{1}, \mathrm{n}_{2}$ are the refractive indices of the superconductor and dielectric materials. The electric field $\mathrm{E}(\mathrm{x})$ in the $\mathrm{m}^{\text {th }}$ unit cell can be written as

$E(x)=\left\{\begin{array}{l}a_{m} e^{-i k_{1}(x-m d)}+b_{m} e^{i k_{2}(x-m d)} ; \quad\left(m d-d_{1}\right)<x<m d \\ c_{m} e^{-i k_{2}\left(x-m d+d_{1}\right)}+d_{m} e^{i k_{2}\left(x-m d+d_{1}\right)} ;(m-1) d<x<\left(m d-d_{1}\right),\end{array}\right.$

(2) where 


$$
\begin{aligned}
& \mathrm{k}_{1}=\left[\left(\frac{\omega}{\mathrm{c}} \mathrm{n}_{1}\right)^{2}-\beta^{2}\right]^{1 / 2}=\frac{\mathrm{n}_{\mathrm{d}} \omega}{\mathrm{c}} \cos \theta_{1} \\
& \mathrm{k}_{2}=\left[\left(\frac{\omega}{\mathrm{c}} \mathrm{n}_{2}\right)^{2}-\beta^{2}\right]^{1 / 2}=\frac{\mathrm{n}_{\mathrm{s}} \omega}{\mathrm{c}} \cos \theta_{2}
\end{aligned}
$$

For the superconductor, the index of refraction can be described on the basis of the conventional two fluid models [13-14]. The two-fluid model is used to describe the electromagnetic behavior of superconductor at non-zero temperature. Normally, the conductivity of the superconductor is complex. With some approximations (lossless), the complex conductivity of a superconductor approximates to $\sigma(\omega)=\frac{-\mathrm{ie}^{2} \mathrm{n}_{\mathrm{s}}}{\mathrm{m} \omega}$

(4) only when the conduction $n_{n} \tau<i_{s} / \omega$ is satisfied. Where $\sigma(\omega)$ is the conductivity of the superconductor, e and $\mathrm{m}$ are the charge and mass of electron respectively, $\mathrm{n}_{\mathrm{s}}$ is the density of electron and $\omega$ is the frequency of external electromagnetic wave. The approximation condition can be found in Ref. [15]. The conductivity equation (4) can be expressed in terms of London-penetration depths $\lambda_{\mathrm{L}}$, since

$$
\begin{aligned}
& \lambda_{\mathrm{L}}^{2}=\frac{\mathrm{m}}{\mu_{0} \mathrm{n}_{\mathrm{s}} \mathrm{e}^{2}} \\
& \sigma(\omega) \approx-\frac{\mathrm{i}}{\mu_{0} \omega \lambda_{\mathrm{L}}^{2}}
\end{aligned}
$$

So that

From the Gorter-Casimir result $[13,15]$, $\frac{\mathrm{n}_{\mathrm{s}}}{\mathrm{n}_{\mathrm{n}}}=\left(\frac{\mathrm{T}_{\mathrm{c}}}{\mathrm{T}}\right)^{4}-1$ and the London-penetration depth, we obtain

$$
\lambda_{L}(T)=\frac{\lambda_{L}(O)}{\sqrt{1-\left(\frac{T}{T_{c}}\right)^{4}}}
$$

(6)

where the conductivity equation (5b) is temperature dependent. In the superconductor layer, with no external source current and charge, the Maxwell's equation becomes - $\quad \vec{\nabla}^{2} \overrightarrow{\mathrm{B}}+\mathrm{k}_{1}^{2} \overrightarrow{\mathrm{B}}=0$

By substituting equation (5), we have

$$
\mathrm{k}_{1}^{2}=\left(\frac{\omega^{2}}{\mathrm{c}^{2}}-\frac{1}{\lambda_{\mathrm{L}}^{2}}\right)
$$

Then, from Snell's law, the length of the tangential wave vector $\mathrm{k}_{1 \mathrm{x}}$ (parallel to the dielectric superconductor interface) is conserved. That is $\mathrm{k}_{1 \mathrm{y}}=\frac{\omega}{\mathrm{c}} \sin \theta=\beta$, where $\theta$ is the angle of incident (relative to normal of interface) of the electromagnetic wave as vacuum. Then, we have the frequency dependent normal vector, $\mathrm{k}_{1 \mathrm{x}}=\sqrt{\frac{\omega^{2}}{\mathrm{c}^{2}} \cos ^{2} \theta-\frac{1}{\lambda_{\mathrm{L}}^{2}}}=\frac{\omega}{\mathrm{c}} \mathrm{n}_{1}(\omega)$

(9) where

$\mathrm{n}_{1}(\omega)=\sqrt{\cos ^{2} \theta-\frac{\mathrm{c}^{2}}{\omega^{2}} \frac{1}{\lambda_{\mathrm{L}}^{2}}}$.

In the

dielectric layer, with no external source current and charge, the Maxwell's equation becomes $\vec{\nabla}^{2} \vec{B}+k_{2}^{2} \quad \vec{B}=0$

From equation (10) and Snell's law, we have $\mathrm{k}_{2}^{2}=\varepsilon \frac{\omega^{2}}{\mathrm{c}^{2}}$ or

$\mathrm{k}_{2 \mathrm{x}}^{2}=\varepsilon \frac{\omega^{2}}{\mathrm{c}^{2}}-\frac{\omega^{2}}{\mathrm{c}^{2}} \sin ^{2} \theta=\frac{\omega^{2}}{\mathrm{c}^{2}}\left(\varepsilon-\sin ^{2} \theta\right)$

or

$$
\mathrm{k}_{2 \mathrm{x}}=\frac{\omega}{\mathrm{c}} \mathrm{n}_{2}
$$

where

$$
\mathrm{n}_{2}=\sqrt{\varepsilon-\sin ^{2} \theta}
$$

According to transfer matrix method [16-17], the characteristic matrix corresponding to one period is as follows

$$
\mathrm{m}(\mathrm{d})=\left(\begin{array}{ll}
\mathrm{m}_{1,1} & \mathrm{~m}_{1,2} \\
\mathrm{~m}_{2,1} & \mathrm{~m}_{2,2}
\end{array}\right)
$$

Where

$\mathrm{m}_{1,1}=\cos \left(\mathrm{k}_{1 \mathrm{x}} \mathrm{d}_{1}\right) \cos \left(\mathrm{k}_{2 \mathrm{x}} \mathrm{d}_{2}\right)-\frac{\mathrm{p}_{2}}{\mathrm{p}_{1}} \sin \left(\mathrm{k}_{1 \mathrm{x}} \mathrm{d}_{1}\right) \sin \left(\mathrm{k}_{2 \mathrm{x}} \mathrm{d}_{2}\right)$ $m_{1,2}=\frac{i}{p_{2}} \cos \left(k_{1 x} d_{1}\right) \sin \left(k_{2 x} d_{2}\right)+\frac{i}{p_{1}} \sin \left(k_{1 x} d_{1}\right) \cos \left(k_{2 x} d_{2}\right)$ $m_{2,1}=i p_{1} \sin \left(k_{1 x} d_{1}\right) \cos \left(k_{2 x} d_{2}\right)+p_{2} \cos \left(k_{1 x} d_{1}\right) \sin \left(k_{2 x} d_{2}\right)$ 
superconductor/dielectric photonic crystal are related

$\mathrm{m}_{2,2}=\cos \left(\mathrm{k}_{1 \mathrm{x}} \mathrm{d}_{1}\right) \cos \left(\mathrm{k}_{2 \mathrm{x}} \mathrm{d}_{2}\right)-\frac{\mathrm{p}_{1}}{\mathrm{p}_{2}} \sin \left(\mathrm{k}_{1 \mathrm{x}} \mathrm{d}_{1}\right) \sin \left(\mathrm{k}_{2 \mathrm{x}} \mathrm{d}_{2}\right)$

where

$\mathrm{k}_{\mathrm{i}}=\frac{\omega}{\mathrm{c}} \mathrm{n}_{\mathrm{i}} \cos \theta_{\mathrm{i}}$ and $\mathrm{p}_{\mathrm{i}}=\frac{\mathrm{n}_{\mathrm{i}}}{\mathrm{Z}_{0}} \cos \theta_{\mathrm{i}}(\mathrm{TE}), \quad \mathrm{p}_{\mathrm{i}}=\frac{1}{\mathrm{Z}_{0} \mathrm{n}_{\mathrm{i}}} \cos \theta_{\mathrm{i}}(\mathrm{TM})$

with $\mathrm{i}=1,2$ and impedance $\mathrm{Z}_{0}\left(=\sqrt{\mu_{0}} / \sqrt{\varepsilon_{0}}\right)$ of

free space. The total characteristic matrix for the $\mathrm{N}$ period structure can be obtained, $\mathbf{M}(\mathrm{Nd})=[\mathrm{m}(\mathrm{d})]^{\mathrm{N}}=\left(\begin{array}{ll}\mathrm{M}_{1,1} & \mathbf{M}_{1,2} \\ \mathbf{M}_{2,1} & \mathbf{M}_{2,2}\end{array}\right)$

(13) where $\mathrm{M}_{1,1}=\mathrm{m}_{11} \mathrm{U}_{\mathrm{N}-1}-\mathrm{U}_{\mathrm{N}-2}, \mathrm{M}_{1,2}=\mathrm{m}_{12}$ $\mathrm{U}_{\mathrm{N}-1} \quad \mathrm{M}_{2,1}=\mathrm{m}_{21} \mathrm{U}_{\mathrm{N}-1}, \quad \mathrm{M}_{2,2}=\mathrm{m}_{22} \mathrm{U}_{\mathrm{N}-1}-\mathrm{U}_{\mathrm{N}-2}$ $\mathrm{m}_{\mathrm{i}, \mathrm{j}}$ are the characteristic matrix of equation (13) and $\mathrm{U}_{\mathrm{N}}$ is the Chebyshev polynomial of second kind defined by

$$
\mathrm{U}_{\mathrm{N}}=\frac{\sin [(\mathrm{N}+1) \mathrm{K}(\omega) \mathrm{d}]}{\sin (\mathrm{K}(\omega) \mathrm{d})}
$$

In order to calculate the transmission and reflectance for a periodic multilayered structure, we use the transfer matrix method. Using this, we can obtain the dispersion relation as

$\mathrm{K}(\omega)=\frac{1}{\mathrm{~d}} \cos ^{-1}\left[\cos \left(\mathrm{k}_{1 \mathrm{x}} \mathrm{d}_{1}\right) \cos \left(\mathrm{k}_{2 \mathrm{x}} \mathrm{d}_{2}\right)-\frac{1}{2}\left(\frac{\mathrm{p}_{1}}{\mathrm{p}_{2}}+\frac{\mathrm{p}_{2}}{\mathrm{p}_{1}}\right) \sin \left(\mathrm{k}_{1 \mathrm{x}} \mathrm{d}_{1}\right) \sin \left(\mathrm{k}_{2 \mathrm{x}} \mathrm{d}_{2}\right)\right]$

The reflection and transmission coefficients can be determined and are given by

$$
\begin{aligned}
& \mathrm{r}=\frac{\left(\mathrm{M}_{1,1}+\mathrm{M}_{1,2} \mathrm{p}_{\mathrm{s}}\right) \mathrm{p}_{0}-\left(\mathrm{M}_{2,1}+\mathrm{M}_{2,2} \mathrm{p}_{\mathrm{s}}\right)}{\left(\mathrm{M}_{1,1}+\mathrm{M}_{1,2} \mathrm{p}_{\mathrm{s}}\right) \mathrm{p}_{0}+\left(\mathrm{M}_{2,1}+\mathrm{M}_{2,2} \mathrm{p}_{\mathrm{s}}\right)} \\
& \mathrm{t}=\frac{2 \mathrm{p}_{0}}{\left(\mathrm{M}_{1,1}+\mathrm{M}_{1,2} \mathrm{p}_{\mathrm{s}}\right) \mathrm{p}_{0}+\left(\mathrm{M}_{2,1}+\mathrm{M}_{2,2} \mathrm{p}_{\mathrm{s}}\right)}
\end{aligned}
$$

(16) Here $p_{0}$ and $p_{s}$ are for the first and last medium of the structure, which are given as $\mathrm{p}_{0}=\frac{\mathrm{n}_{0} \cos \theta_{0}}{\mathrm{Z}_{0}}, \mathrm{p}_{\mathrm{s}}=\frac{\mathrm{n}_{\mathrm{s}} \cos \theta_{\mathrm{s}}}{\mathrm{Z}_{0}}(\mathrm{TE})$ and impedance $Z_{0}\left(=\sqrt{\mu_{0}} / \sqrt{\varepsilon_{0}}\right)$ of free space. In this case, $\mathrm{n}_{0}=\mathrm{n}_{\mathrm{s}}=1.0$ for the free space. The reflectance (R) and transmittance (T) for

$$
\text { by } \quad \mathrm{R}=|\mathrm{r}|^{2} \quad \text { and } \mathrm{T}=\frac{\mathrm{p}_{\mathrm{s}}}{\mathrm{p}_{0}}|\mathrm{t}|^{2}
$$

The expressions of the reflectivity for $\mathrm{N}^{\text {th }}$ layers of the structure is given

$$
\left|\mathrm{R}_{\mathrm{N}}\right|^{2}=\frac{\left|\mathbf{M}_{2,1}\right|^{2}}{\left|\mathbf{M}_{2,1}\right|^{2}+\left(\left|\frac{\sin \mathrm{K}(\omega) \mathrm{d}}{\sin \mathrm{NK}(\omega) \mathrm{d}}\right|\right)^{2}}
$$

where $\mathrm{N}$ is the total number of unit cells (i.e. number of pairs of layers).

\section{REFERENCES}

[1]. Huang-Ming Lee and Jong-Ching Wu, "Transmittance spectra in one-dimensional superconductor-dielectric photonic crystal" J. Appl. Phys. 107, 09E149, 2010.

[2]. G.N. Pandey, Khem B. Thapa and S.P. Ojha' "Omni directional reflectance properties of superconductor-dielectric photonic crystal" Optik - International Journal for Light and Electron Optics, Volume 125, Issue 1, Pages 252-256, January 2014.

[3]. Chun-Li Liu, Hai-Feng Zhang, Yu-Qing Chen, "Enlarged the omnidirectional Bragg gap by one-dimensional superconductor-dielectric photonic crystals with ternary Thue-Morse aperiodic structure" Optik - International Journal for Light and Electron Optics, volume 124, Issue 22, Pages 5811-5817, 2013.

[4]. M. Upadhyay, S. K. Awasthi, L. Shiveshwari, P. K. Srivastava, S. P. Ojha, "Thermally Tunable Photonic Filter for WDM Networks Using 1D Superconductor Dielectric Photonic Crystals" Journal of Superconductivity and Novel Magnetism, Volume 28, Issue 8, pp 2275-2280, 2015.

[5]. J.E.G. Wignhoven and W.L. Vos, Science, 281, 802, 1998.

[6]. A. Velev et al., Nature, 389, 447, 1997.

[7]. A. Figolin, Y.A. Godin, I. Vitebsky, Phys. Rev. B, 57, 284, 1998; P. Halevi and F. Ramos-Medieta, "Tunable Photonic Crystals with semiconducting constituents", Phys. Rev. Lett., 85, 1875-1878, August, 2000.

[8]. W.M. Lee, P.M. Hui and D. Stroud, "Propagating photonic modes below the gap in a superconducting composite", Phys. Rev. B, 51, 8634-8637, April, 1995.

[9]. C.H. Raymond Oai and T.C. Auyeing, Phys. Lett. A, 259, 413, 1999; C.H. Raymond Oai, T.C. Anyeung, C.H. Kam and T.K. Lim, Phys. Rev. B, 61, 5920, 2000; F. Abbas, Propagation in a multilayer structure of superconductor and dielectric, Phyica C, 254, 291-306, June, 1995. 
[10]. Y.B. Chen, C. Zhang, Y. Zhu. S. Zhu and N. Ming, Tunable photonic crystals with superconducting constituents, Mat. Lett., 55, 12-16, July, 2002.

[11]. H. Takeda and K. Yoshino,Tunable photonic band schemes in two-dimensional photonic crystals composed of copper oxide hightemperature superconductors, Phys. Rev. B, 67, 245109-6, June, 2003.

[12]. C. J. Wu, Transmission and reflection in a periodic superconductor/dielectric film multilayer structure, J. Electromagnetic wave and Applications, 19, 1991-1996, 2005; C. J. $\mathrm{Wu}$, Transmission and reflection in a periodic superconductor/dielectric film multilayer structure, PIER Symposium-2005, China, Aug., 2005.

[13]. C. Kittel, Introduction to solid state physics, $6^{\text {th }}$ edition, Wiley, New York, 1986; M.
Tinkham, Introduction to Superconductivity, $2^{\text {nd }}$ edition, McGraw Hill, New York, 1996.

[14]. Thesis by R. N. Yaw on Transmission properties of a high critical temperature superconductor/dielectric multilayer photonic band gap, Huntsvill, Alabama, 2002

[15]. F. Abbas, Propagation in a multilayer structure of superconductor and dielectric, Phyica C, 254, 291-306, June, 1995.

[16]. P. Yeh, "Optical waves in layered media", John Willey and Sons, New York, Chapter 6, 1988.

[17]. M. Born and E. Wolf, "Principle of Optics, Pergmon Press, Oxford, 1965. K. Sakoda, "Optical properties of photonic crystals", Springer Verlag Germany, 2001.

International Journal of Engineering Research and Applications (IJERA) is UGC approved Journal with S1. No. 4525, Journal no. 47088. Indexed in Cross Ref, Index Copernicus (ICV 80.82), NASA, Ads, Researcher Id Thomson Reuters, DOAJ.

J P Pandey. " Calculation of Transmittance of Superconductor-Dielectric Periodic Structure."

International Journal of Engineering Research and Applications (IJERA) 7.7 (2017): 30-33. 